\title{
EMERGING PATTERNS IN CROWD STREAMS AND THE AID OF ABM FOR EGRESS MANAGEMENT
}

\author{
F.A. PONZIANI ${ }^{1,2}$, A. TINABURRI ${ }^{1,2} \&$ M. ANGELINO ${ }^{3}$ \\ ${ }^{1}$ Central Direction for prevention and technical safety - Department of firefighters, of the \\ public rescue and civil defence - Ministry of the Interior - Italy. \\ ${ }^{2}$ University of Rome Tor Vergata - Italy. \\ ${ }^{3}$ University of Loughborough - United Kingdom.
}

\begin{abstract}
The aim of this study is to illustrate the advantage of using agent based models (ABM) in order to deepen the understanding of crowd phenomena and help manage design strategies and egress operations on the field.

The crowd stream, recognised as a proper nonlinear system, is in fact able to produce characteristic patterns that interact with the environment in recurring shapes. In particular, the free stream and the multi-leader stream are chosen as two mostly representative patterns that develop under typical conditions in a real life egress environment, so that the ABM engineering may bring an added value also in the development of the environment layout.

The first part of the study describes the features of these emerging patterns in one geometrical enclosure typical of a Mall. Such issues as queuing, clogging generation, paths of crowd spreading are, for instance, the primary goal upon which to compare the modelling. The overall outcomes, in terms of travel times and people fluxes, complete the scene.

The second part of the study describes the connected evolution of these emerging patterns in an environment typical of the landside space in an Airport terminal.

Such issues as people advancement, emotions transport, and change of direction are, for instance, the primary goal for the modelling. The overall outcomes of the travel times and people fluxes are the remaining issues to be assessed.

The comparison between the results coming from the various ABM packages (Evac, Pathfinder) completes the study, showing how a proper choice in modelling can be based depending on the foreseen goal of the design stage.

The procedure developed in this study, then, may be used and form the basis for an advanced design method that can be of practical use both in Fire Safety Engineering and in Egress Management.

Keywords: agent based model, egress, mall, terminal.
\end{abstract}

\section{EMERGING PATTERNS IN COMPLEX SYSTEMS: CROWD STREAMS}

One of the most astonishing phenomena occurring in complex systems is the appearance of patterns that emerge from the interactions arising in the system, due to internal and external constraints. Depending on the system, these patterns may come from some sort of interaction, like chemical or emotional reactions.

The crowd streams represent one such system, where the interactions among the people have the character of both physical and cognitive nature: think of the constraints due to the free space available for moving or due to the emotional states appearing.

Several approaches have been used to understand and model the crowd streams, from Hydraulics to Particle Dynamics to Cybernetics, and the Agent Based Model (ABM) this research is about may 
use a blend of them. Other models may be considered, from Genetic Algorithms to Cellular Automata, but these are not implemented in the ABM packages used in this study.

While the Hydraulics gives an idea of the global flow, the Particle Dynamics gives an idea of the single entities, the Cybernetics gives an idea of the interactions of various nature, the ABM gives an idea of the behaviours of the agents.

Besides strengths and weaknesses of each approach, the effect of nonlinearity is one of the most important to be captured, as can be underlined considering how some nonlinearity from first principles have been represented.

We can mention three models that have been employed in the realm of crowd dynamics: reaction-diffusion, pseudo-chemical kinetics, wave propagation.

In the reaction-diffusion model [1] the parameter that may exhibit changes in time and space is the concentration of some species that, for the crowd streams, may be represented by the density. The temporal variation is determined by reaction terms that may be derived from a potential plus diffusion terms that let the concentration spatially vary. By a proper choice of the potential and of the diffusivity, the states of the system with nonlinear terms can be investigated, exploring the possibilities for steady state, equilibrium, instability and transition.

In the pseudo-chemical kinetics model [2] the reagents that react following rates in a set of reactions are the cognitive states that, for the crowd streams, may be represented by cognitive behaviours, such as emotions, beliefs and actions. By combining proper reactions sets of cognitive states, the dynamic of the system including nonlinear terms can be modelled and various transitions can be analysed in terms of activation, inhibition, and recovery.

In the wave propagation model [3] the signal that is transferred with a velocity of propagation is any feature of the disturbance that, for the crowd streams, may be represented by the density. The variation of the density in time and space includes the nonlinearity of the propagation velocity that is function of the density. By introducing the flow velocity as the ratio of the density to the flux, and considering a functional relation between the flux and the density, it is possible to express the propagation velocity as the variation of the functional relation of the flux with the density. This leads to the fundamental diagram relating density and flow. Higher order effects may also be included, such as diffusion due to non-local interactions expressed via a density gradient, and response time due to the lag expressed via a desired velocity, which the mover accelerates toward in a relaxation time. Further, shock structures can be introduced to fit the jump discontinuities that may appear in some density distribution.

The emerging patterns that can be observed in the crowd streams may appear as clusters of people and waves of density that form and dissolve in time and space, are more or less persistent in the initial shape appeared, define a gradient, mark some direction.

Depending also on the layout of the environment - especially in terms of space available to be filled and narrow sections to be crossed - and the condition of the situation - especially in terms of real or perceived time of safe movement available - these patterns show like queuing, clogging generation, paths of crowd spreading, people advancement, transport of emotions, abrupt change of direction.

\section{ENVIRONMENT LAYOUT: MALL AND AIRPORT SPACES}

The environment layouts considered in this research are typical of multi-service premises and of transport facilities.

Two layouts have been properly designed here to fit the purpose: one for a mall, one for an airport terminal. These layouts have been chosen since various emerging patterns in the crowd streams may show, due to the high number of people who assemble and move inside. 
The mall is composed of one base floor level and a double height ceiling, representing the link of different spaces, with an overall square-like shape in plan. On the two sides at the floor level, four retail shop spaces align in the left while one assembly space, the restrooms and one bar align on the right, with the mall as the connecting space in between. The side spaces are single height, compared with the double height of the mall. One balcony fills the space at single height, protruding into the mall concourse from the line of the shops. Each space on the sides is connected to the mall through one door, the mall is connected to the outside at the front and the back of the floor with three doors on both, the assembly space has two doors and the bar has one door to the outside on the right side of the floor. The total length of the mall space is $32 \mathrm{~m}$, the total width is $32 \mathrm{~m}, 8 \mathrm{~m}$ on the left, $16 \mathrm{~m}$ in the middle, $8 \mathrm{~m}$ on the right, the single height is $4 \mathrm{~m}$ and the double height is $8 \mathrm{~m}$.

The following Fig. 1 show a rendering of the mall from one visualization tool used for the numerical simulation [4], with some surfaces made fictitiously transparent to let see through.

The airport is composed of one base floor level and a single height ceiling, representing the landside space of the terminal, with an overall strip-like shape in plan. On the left side at the floor level, two partially open square spaces connect to a central concourse, while on the slightly narrower right side a couple of linear walls with turning end corners mark the line. There are four doors to the outside, two in the front part of the concourse and one for each end side, while there are four passageways for each square space that partially open to the concourse.

The total length of the airport space is $60 \mathrm{~m}, 32 \mathrm{~m}$ on the left and $28 \mathrm{~m}$ on the right, the total width is $12 \mathrm{~m}$ on the left and $8 \mathrm{~m}$ on the right, the total height is $4 \mathrm{~m}$. The following Fig. 2 show a rendering of the airport.

The dimension of the doors, passageways and exits - essentially the width - can be varied based on the code followed for the design, both for the mall and the airport.

Several dimensions have been considered in this study, but for the aim of reducing an over-control of the flux that could hinder the formation of patterns well away from the exits, the remainder considers sufficiently wide exits to let patterns develop more freely, at the same time letting possible clogging and queuing with high density of people inside the premises.
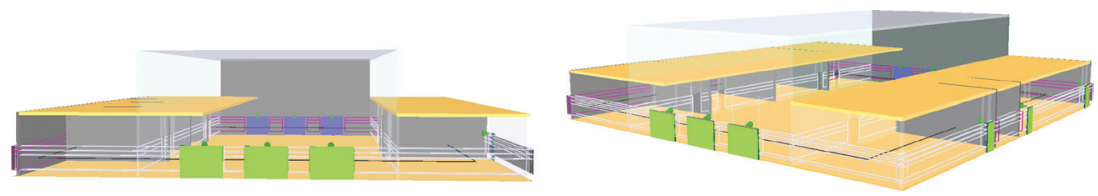

Figure 1: The mall space.
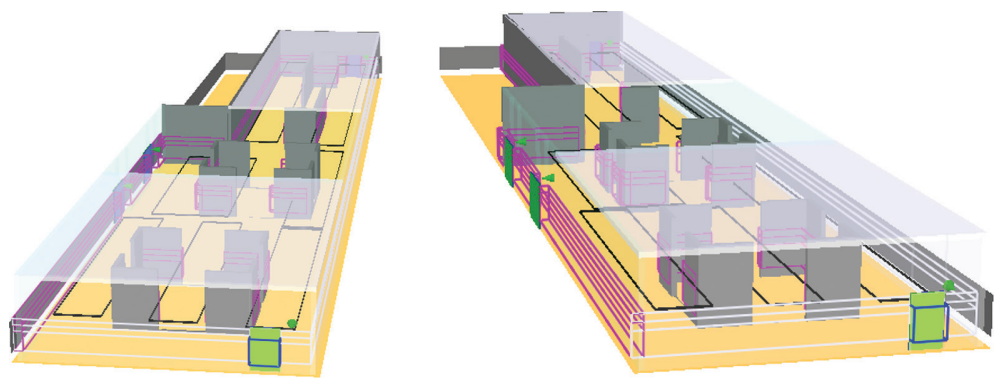

Figure 2: The airport space. 


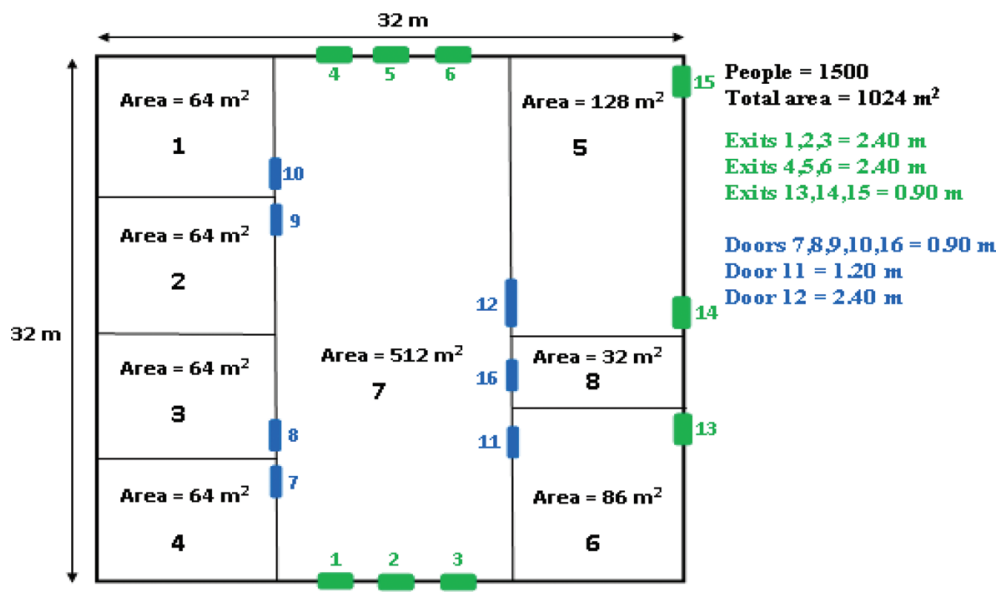

Figure 3: Plan view of the mall.

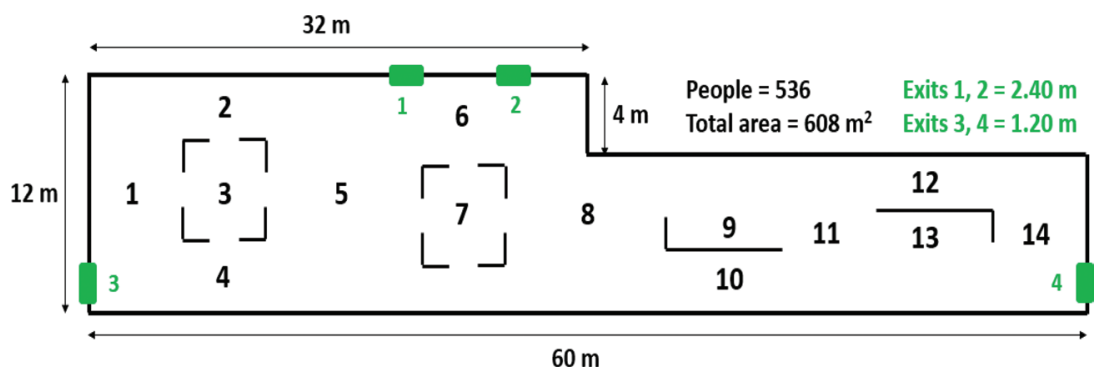

Figure 4: Plan view of the airport.

In order for the emerging patterns of the crowd streams to be coupled to the designed layouts, the virtual environment of the ABM is used here to study what kind of patterns may be represented and how this information may assist in developing proper design strategies and egress operations.

Figures 3 and 4 show the plan of the spaces with some information about spaces and people inside.

\section{AGENT BASED MODELLING: EMERGING PATTERNS AND OVERALL FLUXES}

The principal characteristic of the ABM is to define autonomous entities, called agents, who try to resemble the behaviour of real people in the same situation. Depending on the amount of knowledge of the parameters defining the system, and on the level of the implemented behavioural choices, the agents may wish to simulate some expected or anticipated behaviour in real life. Even if there is a certain variety in the possibilities offered by the ABM packages available to date, the following related to the packages used in this research give an idea of some important aspects: the agents follow some sort of artificial mental mapping, so to say, usually based on some optimization sets of rules for decision making.

The decisions are about the path to be followed from the current position to a target, like doors or exit passageways, considering the priority of reducing the total time of travel, evaluating the formation of queues, assessing the presence of dangerous effects, reacting to stimuli from the environment 
or from other agents, and so on. Two ABM packages have been used for this study: the EVAC (plus FDS) [5] and the Pathfinder [6].

The former is essentially based on the interactions deriving from a Social force model [7] and the latter is essentially based on the interactions from a Steering model [8].

The following Table 1 summarizes the behavioural characteristic assigned to the agents for the numerical simulation of the research.

The assigned names are primarily meant to be internal references to the research for identification, more than fixed behaviours in the ABM packages, and a combination of these names is in fact used sometimes with all the packages depending on the parameters assigned for the simulations.

The numerical simulations refer to the Mall and the Airport layouts designed to highlight the emerging patterns of the crowd stream and have an idea of the overall fluxes of the movement.

In order to capture the essentials for the pattern formation, the runs only consider the movement of the crowd streams in the environment layout with no external source of danger - meaning external to the crowd stream itself - and with practically quick responding time to start.

For future developments of the research, instead, external sources of danger such as fire and unforeseen blockage of exits will be added and the pattern formation will be contrasted with the ones

Table 1: Behavioural characteristics.

\begin{tabular}{|c|c|c|}
\hline \multicolumn{3}{|c|}{ Autonomous Agent } \\
\hline Assignment Identification & ABM Package & Behaviour Highlight \\
\hline Conservative & Evac & $\begin{array}{l}\text { Known door first then the visible ones with } \\
\text { variable known door probabilities }\end{array}$ \\
\hline Conservative 1 & Evac & $\begin{array}{l}\text { Known door first then the visible ones with } \\
\text { fixed known door probabilities set to one for } \\
\text { all exits }\end{array}$ \\
\hline Active plus Herding & Evac & $\begin{array}{l}\text { Visible doors and known doors equal with } \\
\text { fixed known door probabilities set to one for } \\
\text { all exits plus choose the doors that the others } \\
\text { are using if no known doors given }\end{array}$ \\
\hline Active staff plus Herding & Evac & $\begin{array}{l}\text { Visible doors and known doors equal with } \\
\text { fixed known door probabilities set to one for } \\
\text { some exits plus choose the doors that the oth- } \\
\text { ers are using if no known doors given }\end{array}$ \\
\hline $\begin{array}{l}\text { Active staff advanced plus } \\
\text { Herding }\end{array}$ & Evac & $\begin{array}{l}\text { Visible doors and known doors equal with } \\
\text { fixed known door probabilities set to one } \\
\text { for some selected exits plus choose the doors } \\
\text { that the others are using if no known doors } \\
\text { given }\end{array}$ \\
\hline Speedy & Evac & $\begin{array}{l}\text { As Conservative with increased velocities as- } \\
\text { signed to a group }\end{array}$ \\
\hline Steering & Pathfinder & Steering and collision handling \\
\hline Steering plus SFPE & Pathfinder & $\begin{array}{l}\text { Steering and collision handling plus limited } \\
\text { door flow rate }\end{array}$ \\
\hline
\end{tabular}


obtained in the same layout with no external source of danger. Also several distributions of reaction times will be considered.

Moreover, sufficiently total exits width available is considered as a first assessment for the layouts that will be restricted in the future development of the research, in order to contrast with different total times of egress and various design code provisions.

The adoption of a normalized time $\tau=\tau \tau_{\text {current }} / \tau_{\text {end of run }}$ referred to the end time of the run is used for comparing the different simulations and the resulting patterns.

\subsection{Numerical simulations for the Mall layout}

Several runs have been launched varying the characteristics assigned to the agents, ranging from a general knowledge of the various exits present to a preferred knowledge of some particular exit to a slightly increased velocity of movement. For example, the Fig. 5 below shows a sketch of the preference for some exits for the people inside the space where the arrows start from, mimicking a guiding strength of leadership.

The emerging patterns appeared at one third of the runs are showed, for instance, in the following Fig. 6 that compares the patterns emerged by using the Evac ABM (pictures in yellow background), with all the six different behavioural characteristic assigned and the patterns emerged by using also the Pathfinder ABM (pictures in grey background), with two different behavioural characteristic assigned.

The comparison in terms of total time for exiting and usage of some doors is reported in the Figs 7 and 8.

\subsection{Numerical simulations for the Airport layout}

Several runs have been launched varying the characteristics assigned to the agents, in the same way done before. Figure 9 below shows a sketch of the preference for some exits mimicking a guiding leadership:

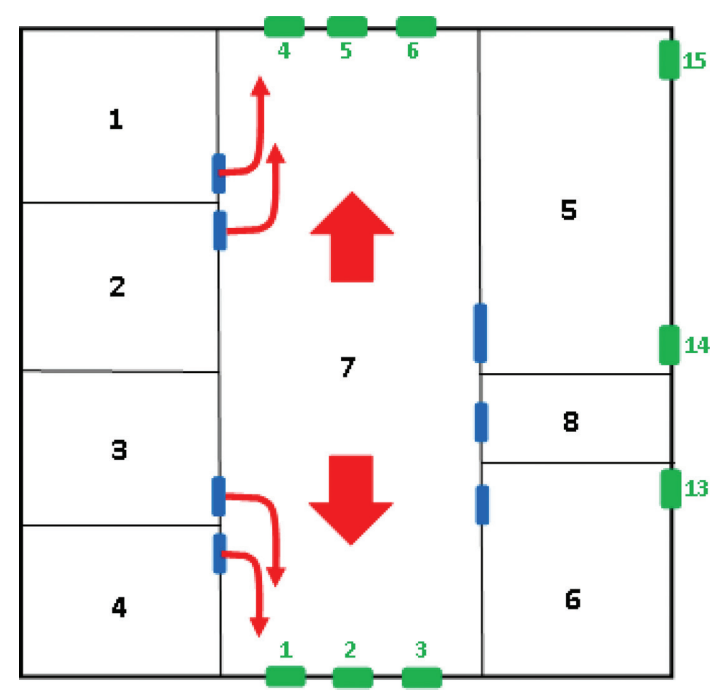

Figure 5: Preference for exiting. 


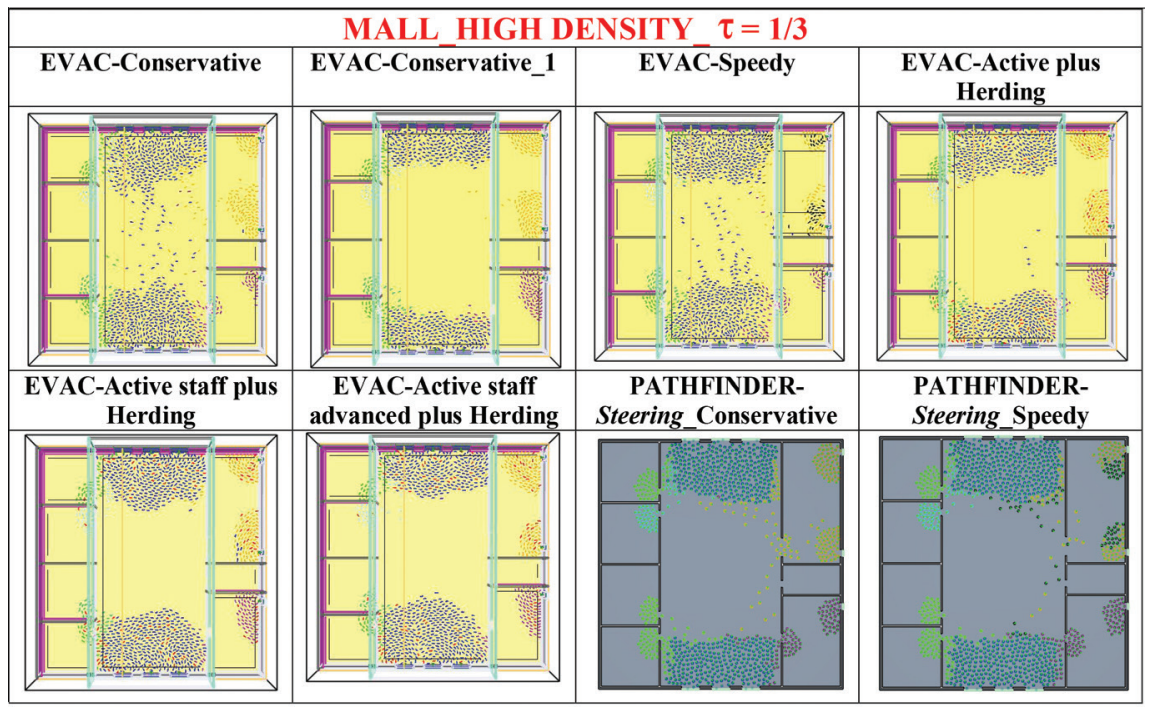

Figure 6: (Evac and Pathfinder): emerging patterns in the mall layout.

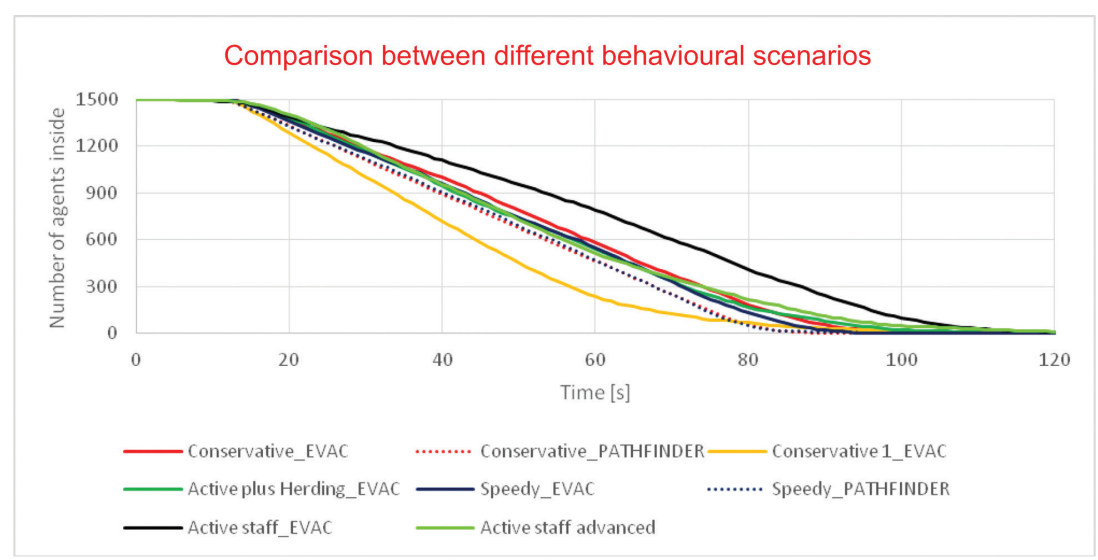

Figure 7: Total time for exiting in the mall layout.
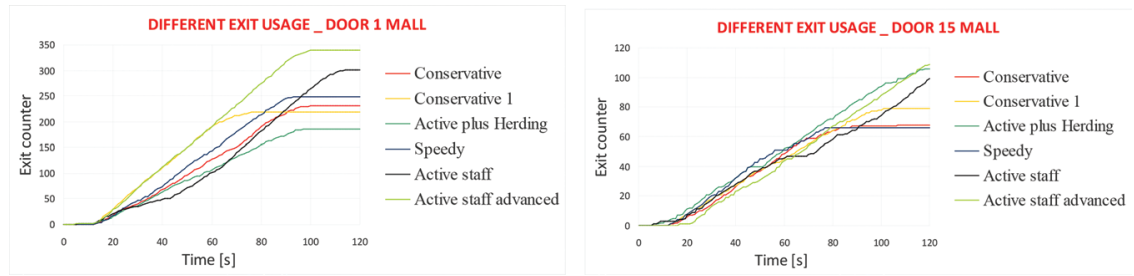

Figure 8: Exit usage trends of two different doors in the mall layout. 
The emerging patterns appeared at one third of the runs are showed, for instance, in the following Fig. 10 that compares the patterns emerged by using the Evac ABM (pictures with purple walls), with four different behavioural characteristics assigned and the patterns emerged by using also the Pathfinder ABM (pictures with grey walls), with two different behavioural characteristic assigned.

The comparison in terms of total time for exiting and usage of some doors is reported in the Figs 11 and 12.

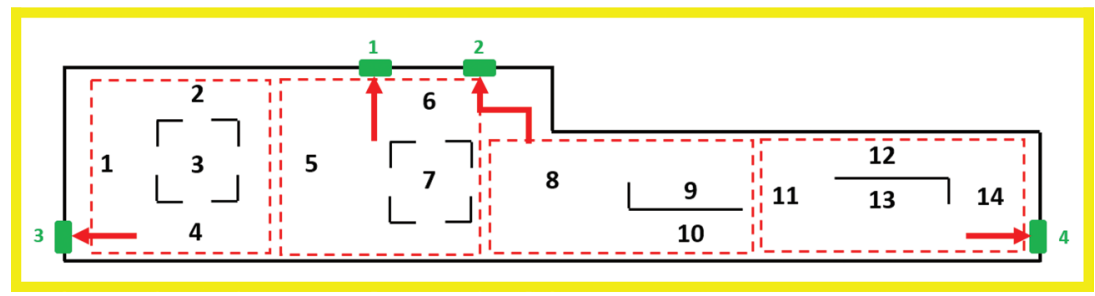

Figure 9: Preference for exiting.

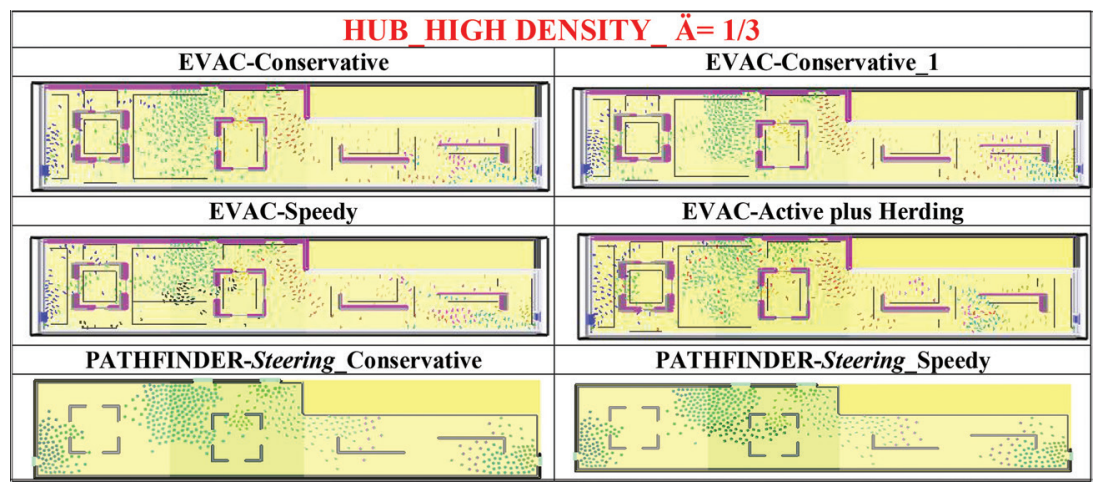

Figure 10: (Evac and Pathfinder) emerging patterns in the airport layout.

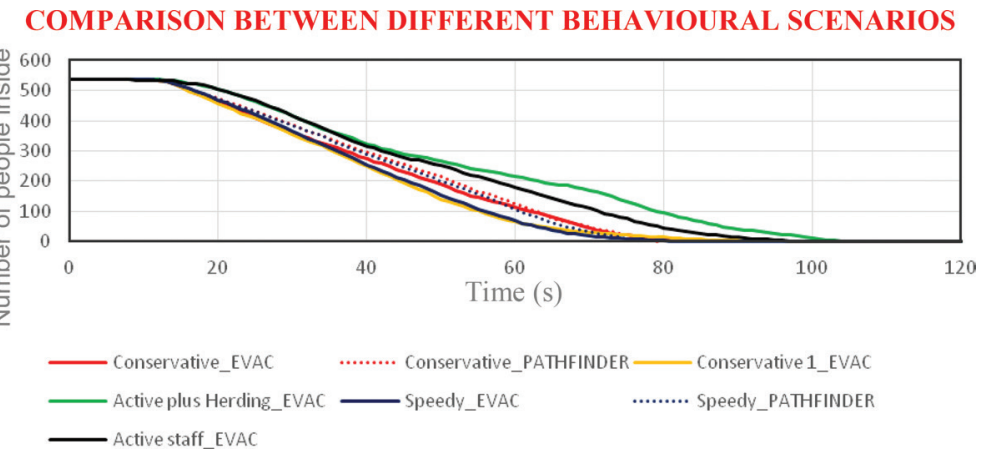

Figure 11: Total time for exiting in the airport layout. 

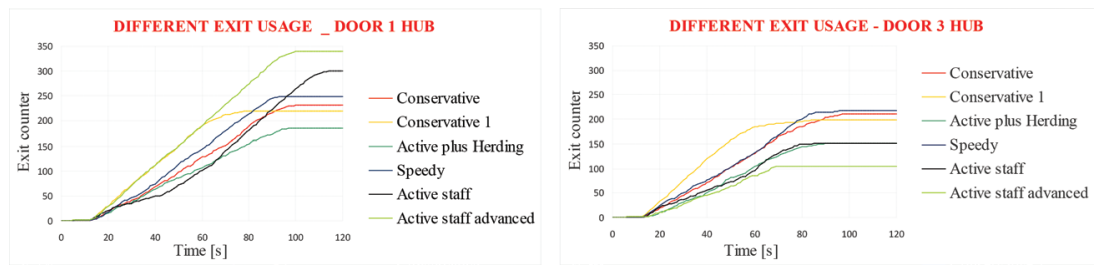

Figure 12: Exit usage trends of two different doors in the airport layout.

MALL_Evacuation at $\mathrm{t}=120 \mathrm{~s}$

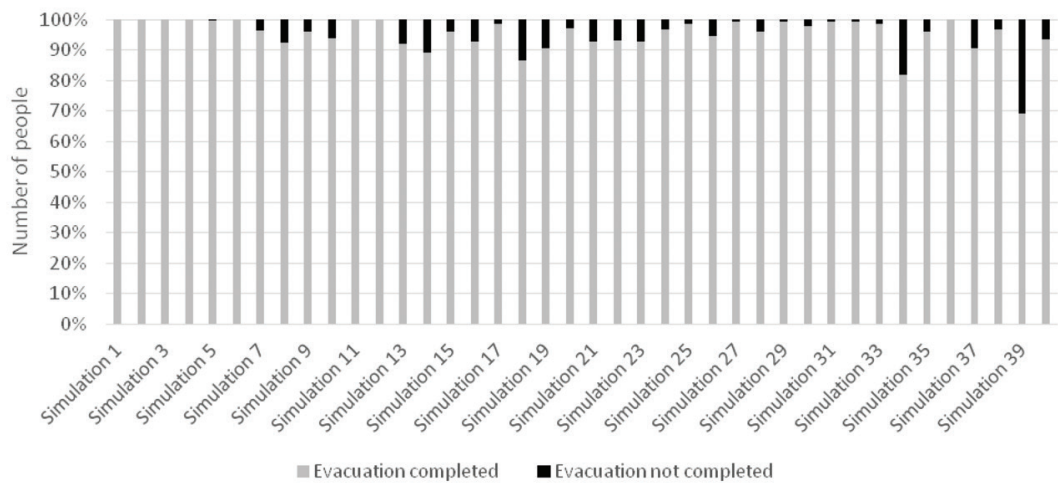

Figure 13: Evacuation state for the mall layout.

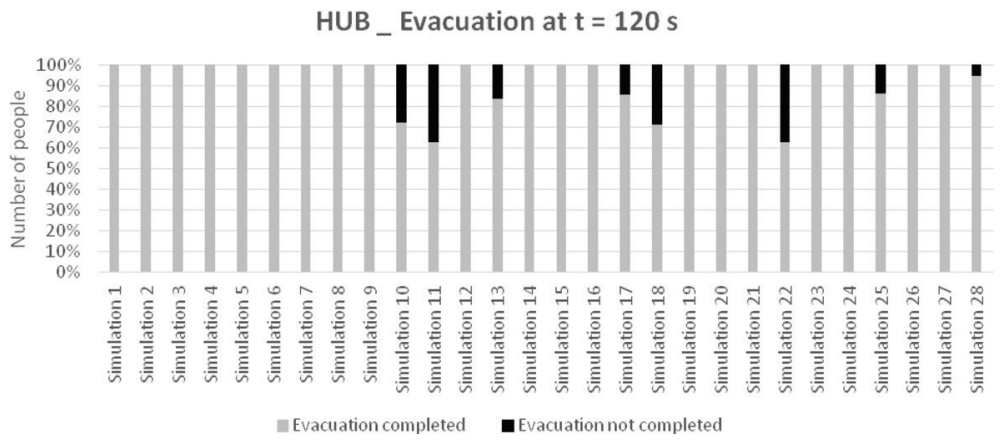

Figure 14: Evacuation state for the airport layout.

\section{CONCLUSION: ADAPTIVE ENGINEERING}

As a general assessment of the designed layout, it is useful to examine the way the simulations have dealt with the total evacuation capacity at the end of the run time scheduled. Figures 13 and 14 show, for the Mall and the Airport layouts, the percentage of people evacuated at the end time of $120 \mathrm{~s}$ scheduled for the runs, considering all the runs performed. 
As can be seen, for the most part all the simulations give complete evacuation at the end time scheduled for the run, for any behaviour assigned to the agents, thus encouraging in the choice made for designing the layouts. Further runs could be performed to improve some characteristics, yet most important is to extend the research to include sources of danger external to the crowd streams.

The patterns generated resemble the emerging ones typical of crowd streams, where the main differences in the shapes are related to the layout of the space - different for the Mall and the Airport - with minor impact of the ABM package used.

A significant impact seems to have the behavioural characteristic assigned to the agents, especially in terms of usage of some exits and consequent slowing or speeding up of the overall crowd streams, with different times to exit.

Further developments of the research might explore the behavioural impact, considering also the internal rules of interactions among the agents and the presence of external sources of danger.

Apart from the practical need of varying the reaction times of the agents and the width of the passageways for applications using different design codes, it is worth-noting to remind the necessity of doing several runs for each type of characteristic implemented for the ABM in order to grasp the dispersions of the data obtained and to take a coefficient of safety in designing a solution.

Better still, experimental observations and databases are hoped in the near future to be available so to balance theory and experience, gaining added value for the design stage also.

In such a way, from Agent Based Modelling a leap could bring to Adaptive Engineering, anticipating the behaviours in a real-life situation through real-time pattern recognition, activating a space layout generation capable of changing in response to the event occurring, considering a way finding from active signals along the paths to guided paths unfolding.

The procedure developed in this study, then, may be used and form the basis for an advanced design method that can be of practical use both in Fire Safety Engineering and in Egress Management.

\section{ACKNOWLEDGEMENTS}

The Authors wish to acknowledge Giordana Gai for her contribution in setting up the simulations.

\section{REFERENCES}

[1] Haken, H., Synergetics. Introduction and Advanced Topics, Springer, 2004.

[2] Adamatzky, A., Dynamics of crowd-minds. Patterns of Irrationality in Emotions, Beliefs and Actions, World Scientific, 2005.

[3] Whitham, G.B., Linear and Nonlinear Waves, John Wiley \& Sons, 1999. http://dx.doi.org/10.1002/9781118032954

[4] Smokeview, NIST Special Publication 1017-1, 2014, Online. http://www.nist.gov/el/fire_ research/fds_smokeview.cfm

[5] Evac (plus FDS), VTT Technical Research Center of Finland, Online. http://www.vtt.fi/fdsevac/

[6] Pathfinder, Thunderhead Engineering, Online. http://thunderheadeng.com/pathfinder.

[7] Helbing, D., Farkas, I. \& Vicsek, T., Simulating dynamical features of escape panic. Nature, 407, pp. 487-490, 2000.

[8] Reynolds, C., Steering behaviours for autonomous characters. Proceeding Game Developers Conference, pp. 763-782, 1999. 